\title{
Neurogenic Bladder Due to Hypoxic-Ischemic Demyelination
}

\author{
Sindhu Ramchandren, MD, David S. Liebeskind, MD \\ From the University of Michigan Health System, Neurology, Ann Arbor, MI (SR); and UCLA Stroke Center-University of California, Los Angeles, CA (DSL).
}

\section{A B S T R A C T}

\section{BACKGROUND AND PURPOSE}

Hypoxia is commonly known to target neuronal cell bodies. Although myelin is a non-infrequent target, posthypoxic demyelination is a rarely described entity. We describe the case of a man who developed neurogenic bladder following a motor vehicle accident.

\section{CASE REPORT}

Following a severe motor-vehicle accident involving massive blood loss, a 46-year-old man developed urinary urgency requiring catheterization, with hyperactive reflexes and bilateral Babinski signs on exam.

\section{RESULTS}

Magnetic resonance imaging of the brain and cervical cord revealed diffuse, isolated white matter signal abnormality in a symmetric, confluent distribution, extending inferiorly into the spinal cord, with changes consistent with bilateral Wallerian degeneration. Serologic and CSF evaluations were normal.

\section{CONCLUSIONS}

Unlike previous reports of hypoxic-ischemic demyelination, our patient lacked any cortical abnormalities, presumably due to isolated white matter changes. This report alerts physicians to the possibility of hypoxic-ischemic demyelination due to global hypoxia-ischemia as an etiologic factor for neurogenic bladder.
Keywords: Hypoxia, ischemia, demyelination, neurogenic bladder, Wallerian degeneration.

Acceptance: Received October 6, 2006, and in revised form May 24, 2007. Accepted for publication June 18, 2007.

Correspondence: Address correspondence to Sindhu Ramchandren, MD, Department of Neurology, University of Michigan at Ann Arbor, 300 North Ingalls St, \#3D06, Ann Arbor, Ml 48109. E-mail: sindhur@med.umich.edu.

Conflict of Interest: The authors have reported no conflicts of interest.

J Neuroimaging 2008;18:198-201. DOI: 10.1111/j.1552-6569.2007.00167.x

\section{Background}

Neurological complications of hypoxia-ischemia can be varied; the most common presentations involve cortical loss resulting in neuropsychological manifestations. We describe a case of neurogenic bladder due to trauma-induced hypoxicischemic demyelination. This is contrary to the usual presentation of hypoxia and ischemia to the central nervous system and highlights the importance of considering this diagnosis in the differential.

\section{Case Report}

A 46-year-old man was seen in the out-patient neurology clinic for evaluation of neurogenic bladder. He was a missionary man in Guam, visiting the United States for an expedited work-up of his problem. His past medical history was significant for a motor-vehicle accident 6 years ago: he was the restrained driver and was involved in a head-on collision, where he suffered massive blood loss and several injuries, including ruptured spleen, rib fractures, and lacerations to his kidneys and liver. $\mathrm{He}$ was in an intensive care unit for several weeks with decreased levels of arousal, during which time he required multiple blood transfusions. He then underwent a prolonged in-patient and out-patient rehabilitation course to return to full motor function.

Weeks to months after that accident, he noted urinary urgency with decreased flow, requiring intermittent catheter- ization. He developed erectile dysfunction two years later. Over the next few years, the urologic problems worsened. He underwent urodynamic testing and was diagnosed with neurogenic bladder, and was referred to us for a neurologic evaluation.

At the time of his evaluation by us, he had intact sensory and motor function and did not require any assistive devices for mobility. However, he required periodic catheterization and occasionally had urinary incontinence. His review of systems was essentially negative: he denied any other neurologic symptoms, including cognitive changes, or experiencing Lhermitte's or Uhthoff's phenomena. He had no other medical or family history of neurodegenerative disease. On examination, he had a normal general examination. Mental status and detailed cranial nerve examination were normal. Motor exam showed normal tone without evidence of atrophy, and strength was $5 / 5$ throughout. Sensory examination was normal except for decreased vibratory perception distally. Gait and coordination were normal. Reflexes were $3+$ throughout, and plantar responses were extensor bilaterally.

Magnetic resonance imaging (MRI) of the brain and cervical spine was obtained urgently, which showed diffuse white matter abnormality at the centrum semiovale and corona radiata with increased signal intensity in a symmetric, confluent distribution (Figs 1 and 2). The white matter signal abnormality extended inferiorly through the medulla, demonstrating changes consistent with bilateral Wallerian degeneration; these changes continued 

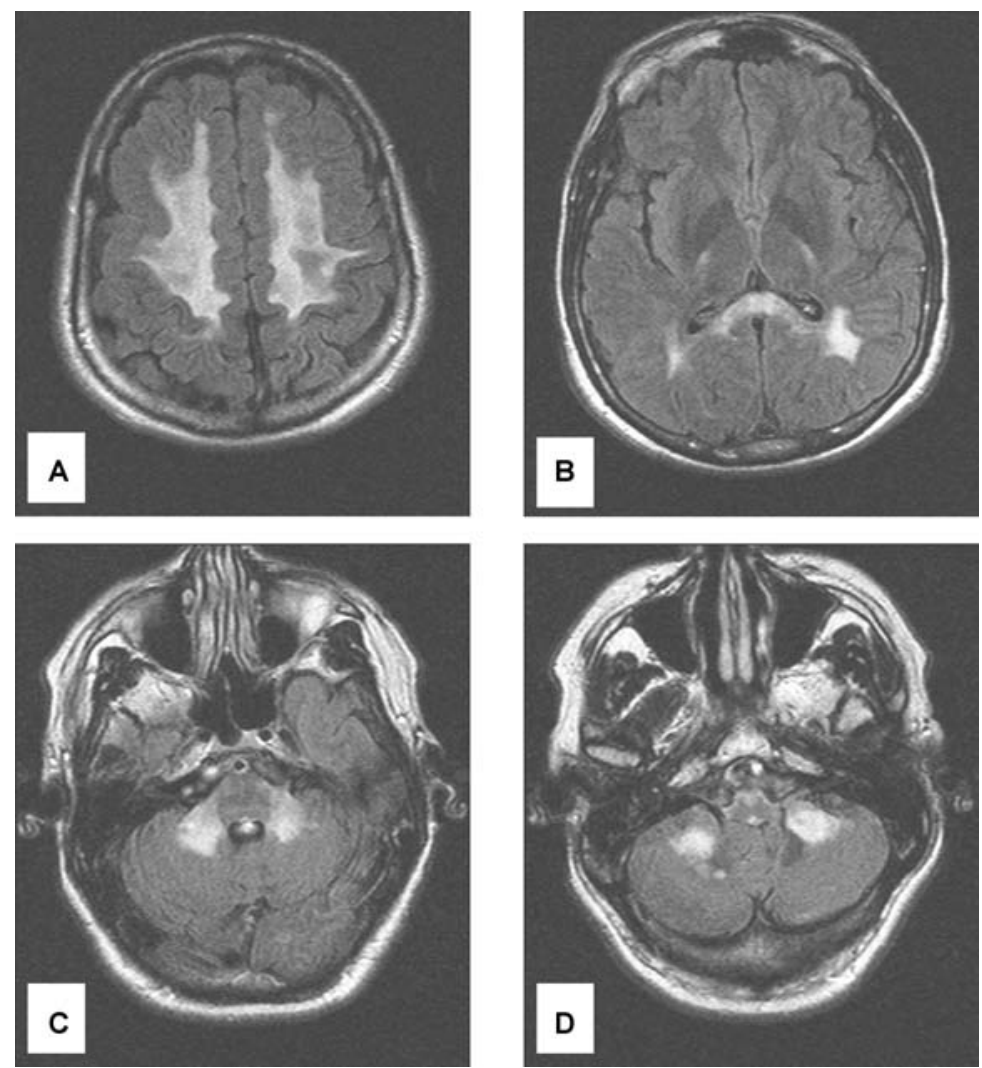

Fig 1. Axial brain MRI, flair: diffuse white matter hyperintensity in a symmetric, confluent distribution, extending from (A) the centrum semiovale and corona radiata (B) corpus collosum (C) pons and (D) the atrophic medulla demonstrating bilateral Wallerian degeneration.
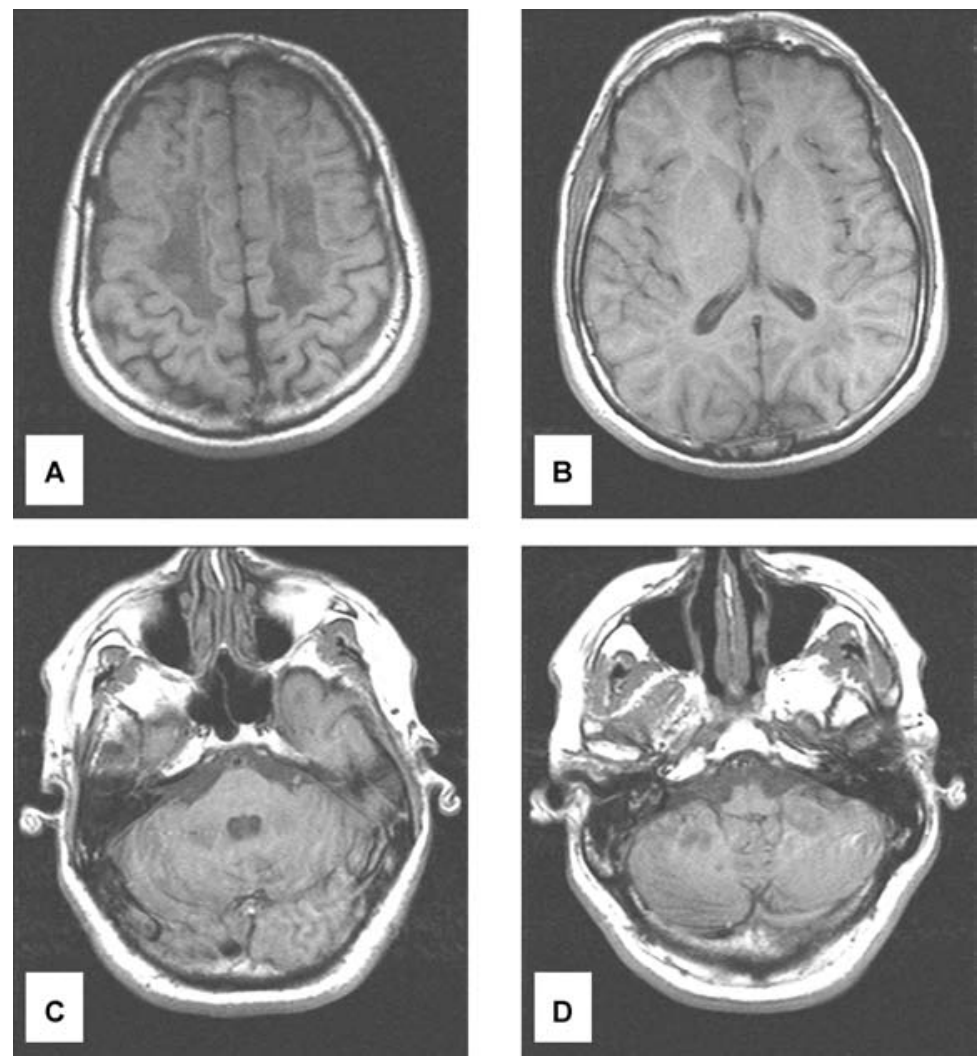

Fig 2. Axial brain MRI, $T 1$ : diffuse white matter $T 1$ hypointensity in a symmetric, confluent distribution, extending from (A) the centrum semiovale and corona radiata (B) corpus collosum (C) pons and (D) the atrophic medulla demonstrating bilateral Wallerian degeneration. 

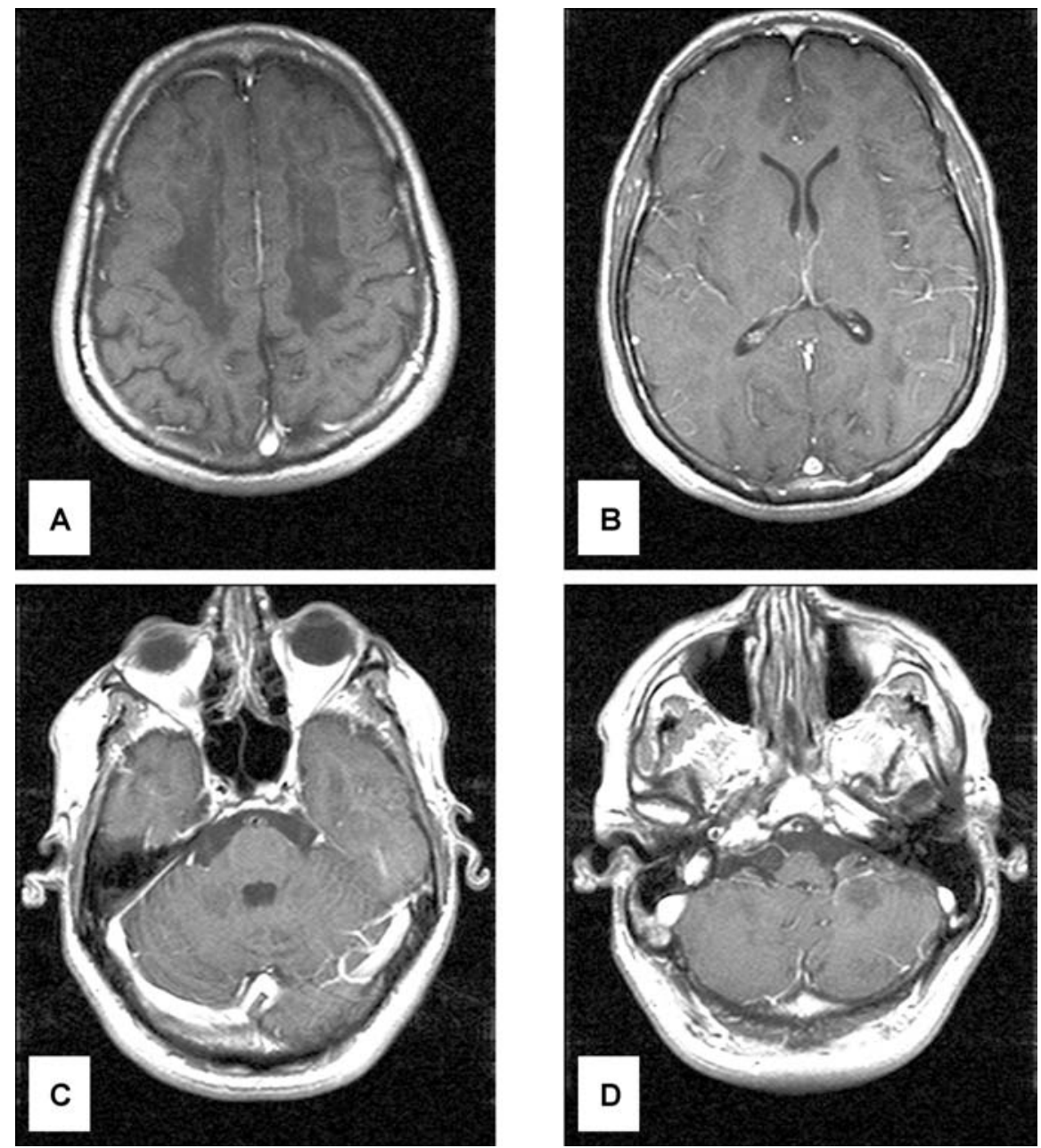

Fig 3. Axial brain MRI, T1 postcontrast: $\mathrm{T} 1$ hypointensity shows no enhancement, in $(\mathrm{A})$ the centrum semiovale and corona radiata (B) corpus collosum (C) pons or (D) the atrophic medulla demonstrating bilateral Wallerian degeneration.

well into the spinal cord. The cortex, including cortical U-fibers, and the putamen, were spared. There were no masses and no pathologic enhancement (Fig 3).

Serologies including B12 and folate levels, RPR, HIV, ANA, $\mathrm{TSH}$, erythrocyte sedimentation rate, ACE level, serum protein electrophoresis, and hepatitis serologies were all normal. His CSF analysis revealed a white count of 1 , red blood cell count of 0 , a glucose level of 62 , and protein level of 40 . The CSF cultures were negative for bacterial or fungal growth; testing for cryptococcal antigen was negative. The PCR for JC virus and CMV were both negative. The CSF protein electrophoresis was normal, with no oligoclonal bands. At the conclusion of our investigation, the patient was diagnosed with posthypoxic demyelination as a cause of his neurogenic bladder.

\section{Discussion}

Posthypoxic demyelination is a rarely described entity. Although hypoxia is commonly known to target neuronal cell bodies, myelin is a non-infrequent target. Original reports of hypoxic-ischemic demyelination frequently found associated changes in the gray matter as well, albeit relatively less common. ${ }^{1}$ Several later reports documented an almost exclusive effect of hypoxia-ischemia on white matter. ${ }^{2,3}$ One report found reduced levels of arylsulfatase $\mathrm{A}$, and proposed that the consequent myelin metabolic dysfunction could predispose vulnerable individuals to delayed demyelination after hypoxia $;^{4}$ similar cases have subsequently been reported. ${ }^{5,6}$ We did not assess our patient's fatty acids or arylsulfatase levels, especially since there have been reports of wide-spread demyelination even in the absence of arylsulfatase deficiency. The authors of one such report proposed necrosis of myelin in the border zones of the white matter with its widely spaced linear arterioles, whereas better vascular supply preserved the gray matter. $^{7}$

The clinical presentation and neurologic examination of our patient were notable for the upper motor neuron findings, no sensory level, and lack of cortical signs, suggestive of a diffuse white matter process. The differential diagnosis for the abnormal neuroimaging findings was initially broad, including traumatic etiologies such as diffuse demyelination secondary to significant trauma-induced hypotension and is- 
chemia, infectious etiologies such as progressive multifocal leukoencephalopathy (PML) due to JC virus, or HIV or neurosyphilis acquired during his multiple blood transfusions, vitamin deficiencies such as B12 deficiency causing subacute combined degeneration, endocrinopathies such as Hashimoto's encephalitis (although the presentation was atypical), vasculitis, and toxin exposure.

Our conclusion at the end of our investigation, which ruled out all the other etiologies in our differential, was that our patient had suffered hypoxic-ischemic injury to his brain and had subsequent white matter demyelination and Wallerian degeneration of his cord. Due to his work as a missionary, he left the country a few days after his visit to our clinic, and we have not had success in obtaining follow-up information. This report alerts physicians to the possibility of hypoxic-ischemic demyelination due to global hypoxia-ischemia as an etiologic factor for neurogenic bladder.

\section{References}

1. Ginsberg MD, Hedley-White T, Richardon EP. Hypoxic-ischemic leukoencephalopathy in man. Arch neurol 1976;33:5-14.

2. Arbelaez A, Castillo M, Mukherji SK. Diffusion-weighted imaging of global cerebral anoxia. Am J Neuroradiol 1999;20:999-1007.

3. Pantoni L, Garcia JH, Gutierrez JA. Cerebral white matter is highly vulnerable to ischemia. Stroke 1996;27:1641-1647.

4. Weinberger LM, Schmidley JW, Schafer IA, Raghavan S. Delayed postanoxic demyelination and arylsulfatase-A pseudodeficiency. Neurology 1994;44:152-154.

5. Gottfried JA, Mayer SA, Shungu DC, Chang Y, Duyn JH. Delayed posthypoxic demyelination: association with arlysulfatase A deficiency and lactic acidosis on proton MR spectroscopy. Neurology 1997;49:1400-1404.

6. Miura S, Ohyagi Y, Ohno M, et al. A patient with delayed posthypoxic demyelination: a case report of hyperbaric oxygen treatment. Clin Neurol Neurosurg 2002;104:311-314.

7. Heckmann JG, Erbguth F, Neundorfer B. Delayed postanoxic demyelination registry. Neurology 1998;51:1235-1236. 